\title{
FLAT SPACE CONGRUENCES OF ORDER ONE IN $[n]$
}

\author{
BY \\ EDWIN J. PURCELL
}

By an $[n-k]$-congruence of order one in $[n], k<n$, is meant an algebraic $\infty^{k}$-system of $[n-k]$ 's in $n$-dimensional projective space such that one and only one $[n-k]$ of the system passes through an arbitrary point of $[n]$. A point on more than one $[n-k]$ of the congruence is called a fundamental point. A fundamental point fails to determine any $[n-k]$ of the congruence.

Although some special cases of flat space congruences have been studied, there is no general theory in the literature $\left.{ }^{1}\right)$. Line congruences of order one in [3] were investigated by $E$. Kummer( $\left.{ }^{2}\right)$ and this was completed by R. Sturm( $\left.{ }^{3}\right)$. G. Marletta $\left({ }^{4,5}\right)$ classified line congruences of order one in [4] but his methods were not suitable for extension to $[n]$. Some line congruences in [5] were discussed by M. Sgroi $\left({ }^{6}\right)$. G. Aprile $\left({ }^{7}\right)$ collected the known examples of line congruences of order one in $[n]$; these are all special cases of the more general congruences defined in the present paper and it will be shown in $\$ 8$ that his construction for a line congruence in $[n]$ with irreducible locus of fundamental points is a restricted case of one of ours $\left({ }^{8}\right)$.

Probably not all possible flat space congruences of order one are included in what follows. The line congruence in [4] consisting of the trisecants of the $F^{4}$, projection of the Veronese surface in [5], and the two line congruences in [5] consisting of the bisecants of a ruled $F^{4}$ or of the bisecants of the del Pezzo surface, do not appear to belong to our class of congruences.

1. Classification. On each $[n-k]$ of our $[n-k]$-congruences of order one

Presented to the Society, November 28, 1942; received by the editors April 6, 1942, and, in revised form, January $6,1943$.

(1) Encyklopädie der Mathematischen Wissenschaften vol. III C 7, p. 964.

(2) E. Kummer, Über die algebraischen Strahlensysteme, in besondere die der ersten und zweiten Ordnung, Berlin, Abhandlungen der Preussischen Akademie der Wissenschaften, 1866.

$\left.{ }^{3}\right)$ R. Sturm, Die Gebilde ersten und zweiten Grades der Liniengeometrie in synthetischer Behandlung, Leipzig, vol. 2, 1893.

(4) G. Marletta, Sui complessi di rette del primo ordine dello spazio a quattro dimensioni, Rend. Circ. Mat. Palermo vol. 28 (1909).

(5) Marletta, Sopra i complessi di rette d'ordine uno dell $S_{4}$, Atti Accademia Gioenia Catania, (5) vol. 3 (1909).

(6) M. Sgroi, Sui 4-complessi di rette d'ordine uno nell' $S_{5}$, Atti Accademia Gioenia Catania (5) vol. 15 (1925).

(7) G. Aprile, Giornale di Matematica vol. 70 (1932) pp. 196-216.

${ }^{8}$ ) After the present paper had been sent to the editor, I received from Professor Virgil Snyder an offprint of his Cremona involutions belonging to the Bordiga surface in [4], Revista de Tucumán (A) vol. 2 (1941) pp. 203-210, in which he describes a line congruence of order one in $[n]$ consisting of the $(n-1)$-secants of the generalized Bordiga. This is type $(n-1)_{n}$ in my classification. 
in $[n]$ there is a $V_{n-k-1}^{k}$, all of whose points are fundamental. ( $V_{0}^{k}$ means $k$ points.) This variety may be irreducible or composite. In the latter case the dimension of each component is $n-k-1$ while their orders may be any positive integers $A_{1}, A_{2}, \cdots, A_{w}$, whose sum is $k$.

The type symbol $\left(A_{1} A_{2} A_{3} \cdots A_{w}\right)_{n}$ will be used to indicate an $[n-k]$ congruence of order one in $[n]$ whose generic $[n-k]$ has on it $w$ distinct fundamental varieties of orders $A_{1}, A_{2}, \cdots, A_{w}$, respectively. When the meaning of the type symbol becomes completely evident ( $\$ 4)$, it will be seen that permutation of the $A_{i}$ in the type symbol changes the type. Therefore there are $2^{k-1}$ types of $[n-k]$-congruences of order one in $[n]$, for which the fundamental loci on a generic $[n-k]$ are all distinct.

Since some or all of the fundamental varieties on a generic $[n-k]$ of the congruence may be coincident $(\$ 6)$, we divide $[n-k]$-congruences of order one in $[n]$ into those for which these components are all distinct and those for which some or all are coincident. Until $\$ 6$, congruences in the former category will occupy us.

2. Fixed base. The equations

$$
\begin{aligned}
& \lambda_{1} x_{11}+\cdots+\lambda_{r+1} x_{1 r+1}=0, \\
& \cdot \cdot \cdot \cdot \cdot \cdot \cdot \cdot \cdot \cdot \cdot \cdot \lambda_{r+1} x_{r+1}=0 \\
& \lambda_{1} x_{r 1}+\cdots+
\end{aligned}
$$

in which the $\lambda_{j}$ are parameters and the $x_{i j}$ are linear forms in the homogeneous projective point coordinates $x_{0}, x_{1}, \cdots, x_{n}$ of $[n]$, having arbitrary constant coefficients, represent an $\infty^{r}$-system of $[n-r]$ 's in $[n]$.

A necessary and sufficient condition for an $[n-r]$ of this system to pass through an arbitrary point $P$ of $[n]$ is

$$
\begin{gathered}
\lambda_{1} p_{11}+\cdots+\lambda_{r+1} p_{1 r+1}=0, \\
\cdot \ldots+\lambda_{r+1} p_{r+1}=0, \\
\lambda_{1} p_{r 1}+\cdots \cdot
\end{gathered}
$$

where $p_{i j}$ is the result of substituting the coordinates of $P$ in $x_{i j}$.

The symbol

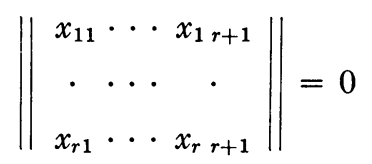

means $r+1$ simultaneous equations $X_{h}=0(h=1,2, \cdots, r+1)$, where $X_{h}$ is $(-1)^{h+1}$ times the determinant formed from the matrix in (2.3) by omitting the $k$ th column.

Denoting by $P_{h}$ the result of substituting $p_{i j}$ for $x_{i j}$ in $X_{h}$, we have from $(2.2)$ 


$$
\lambda_{1}: \lambda_{2}: \cdots: \lambda_{r+1}=P_{1}: P_{2}: \cdots: P_{r+1}
$$

Therefore

$$
\begin{gathered}
P_{1} x_{11}+\cdots+P_{r+1} x_{1 r+1}=0 \\
\cdots \cdots+P_{r+1} x_{r+1}=0 \\
P_{1} x_{r 1}+\cdots+
\end{gathered}
$$

are the equations of the $[n-r]$ of system (2.1) through point $P$.

Now a necessary condition for any other point $Q$ to lie on $(2.4)$, the $[n-r]$ through $P$, is

$$
\begin{gathered}
P_{1} q_{11}+\ldots+P_{r+1} q_{1 r+1}=0, \\
\ldots \ldots+P_{r+1} q_{r+1}=0, \\
P_{1} q_{r 1}+\cdots .
\end{gathered}
$$

where the $q_{i j}$ are obtained by substituting the coordinates of $Q$ in the $x_{i j}$. From which $P_{1}: P_{2}: \cdots: P_{r+1}=Q_{1}: Q_{2}: \cdots: Q_{r+1}$. Thus every point on (2.4) determines that same $[n-r]$, and the ratios $P_{1}: P_{2}: \cdots: P_{r+1}$ are unique for the $[n-r]$ of the system (2.1) passing through $P$.

Since (2.4) defines one $[n-r]$ through an arbitrary point $P$ of $[n]$ and every point of (2.4) determines that same $[n-r]$, it follows that the totality of $[n-r]$ 's given by (2.4) for all points $P$ of $[n]$ form an $[n-r]$-congruence of order one in $[n]$.

(2.3) is a determinantal locus $\left({ }^{9}\right)$. Since it will appear that (2.3) serves as a carrier or base for flat space congruences of order one, (2.3) will be called a fixed base.

Indicate the fixed base (2.3) by $A_{1}$. It is a variety of dimension $n-2$ and order $C_{r+1,2}\left({ }^{10}\right)$. Degenerate cases, for which the order of the fixed base is lower, will be treated in a separate note.

The points of $\mathcal{A}_{1}$ are fundamental points for the $[n-r]$-congruence of order one in $[n]$ whose generic $[n-r]$ is given by (2.4).

For a general point $P$ not on $A_{1}$, at least one of the $P_{j}(j=1,2, \cdots, r+1)$ is different from zero. For convenience, let $P_{r+1} \neq 0$. If all the other $P_{j}$ are zero, it is obvious that the intersection of the $[n-r]$ through $P$, given by (2.4), with the fixed base (2.3), is the same as the intersection of (2.4) with

$$
X_{r+1} \equiv\left|\begin{array}{ccc}
x_{11} & \cdots & x_{1 r} \\
\cdot & \cdots & \cdot \\
x_{r 1} & \cdots & x_{r r}
\end{array}\right|=0
$$

If some other $P_{j}$, say $P_{i}$, is also different from zero, (2.6) can be rewritten

(') T. G. Room, The geometry of determinantal loci, Cambridge, 1938, p. 33.

$\left({ }^{10}\right)$ Room, loc. cit. pp. 34, 43. 


$$
\frac{1}{P_{i}}\left|\begin{array}{cc}
x_{11} \cdots x_{1-1}\left(P_{1} x_{11}+\cdots+P_{r} x_{1 r}\right) x_{1+1} \cdots x_{1 r} \\
\cdots & \cdot \\
x_{r 1} \cdots x_{r-1}\left(P_{1} x_{r 1}+\cdots+P_{r} x_{r r}\right) x_{r i+1} \cdots x_{r r}
\end{array}\right|=0 .
$$

Using (2.4), this becomes

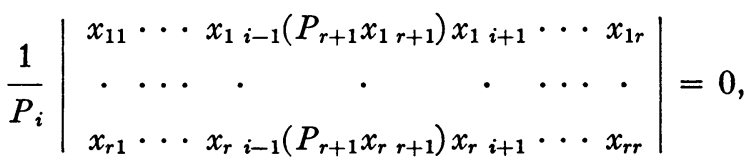

which is $P_{r+1} X_{i} / P_{i}=0$.

Since the selection of $P_{r+1}$ as the nonzero $P_{j}$ was arbitrary, it follows that the intersection of the $[n-r]$ through $P$, given by (2.4), with the fixed base (2.3), is the same as the intersection of (2.4) with any $X_{j}=0$ for which $P_{j} \neq 0$.

This intersection is a determinantal primal in the space $[n-r]$ through $P$ and is of order $r$. Its equation is formed by imposing the $r$ linear conditions (2.4) on the running coordinates $x_{0}, x_{1}, \cdots, x_{n}$ in each element $x_{i j}$ of $X_{j}=0$.

In the fixed base (2.3), let $x_{i j} \equiv \sum_{k=0}^{n} a_{i j k} x_{i}$, where $a_{i j k}$ are constants. Equations (2.1) may be rewritten

$$
\begin{aligned}
& \lambda_{10} x_{0}+\cdots+\lambda_{1 n} x_{n}=0, \\
& \cdot \cdots+\lambda_{r n} x_{n}=0, \\
& \lambda_{r 0} x_{0}+\cdots \cdot
\end{aligned}
$$

where $\lambda_{i k} \equiv \sum_{j=1}^{r+1} \lambda_{j} a_{i j k}$. In general, for each set of values $\lambda_{i}, \cdots, \lambda_{r+1}$, these equations are independent and represent an $[n-r]$ of the congruence based on (2.3). A necessary and sufficient condition for equations (2.7) to be dependent is that the rank of the matrix

$$
\left\|\begin{array}{c}
\lambda_{10} \cdots \lambda_{1 n} \\
\cdots \\
\lambda_{r 0} \cdots \lambda_{r n}
\end{array}\right\|
$$

be $m(m<r)$. In such event, (2.8) represents a variety $\Lambda$ of dimension( $\left.{ }^{11}\right)$ $r-m(n+1-r+m)$ in a space $[r]$ whose generic point has coordinates $\left(\lambda_{1}, \cdots, \lambda_{r+1}\right)$. The order of $\Lambda$ is given by a formula to Segre $\left({ }^{12}\right)$. To each point of $\Lambda$ corresponds a set of values $\lambda_{1}, \cdots, \lambda_{r+1}$ which determine in (2.1), not an $[n-r]$, but an $[n-m]$.

For example, the type (3) $)_{4}$ line congruence of order one in [4] consists of the trisecants of a Bordiga sextic surface. This surface is the fixed base $\mathcal{A}_{1}$, all of whose points are fundamental. But there are 10 planes in [4], each of which cuts the Bordiga in a cubic curve. Through a general point of such a

(11) Room, loc. cit. p. 36.

(12) Room, loc. cit. p. 42. 
plane a pencil of lines can be drawn, each of which is a trisecant of the Bordiga surface. The equations of the 10 planes are obtained by substituting in (2.1) values of $\lambda_{1}, \cdots, \lambda_{r+1}$, which make the rank of matrix (2.8) be $m=2$.

Let $t_{i_{1} i_{2}} \ldots i_{r}=t_{(i)}$ be the $r$-row minors of the matrix (2.8). These minors $t_{(i)}$ are forms in $\lambda_{1}, \cdots, \lambda_{r+1}$. An $[n-r]$ with coordinates $\bar{t}_{i_{1} i_{2}} \ldots i_{r}=\bar{t}_{(i)}$ belongs to the congruence if and only if its coordinates $\bar{t}_{(i)}$ satisfy all homogeneous algebraic relations which are satisfied identically by the $t_{(i)}$; that is, if and only if $F\left(\cdots, t_{i_{1} i_{2}} \ldots i_{r}, \cdots\right)=0$ (identically in $\left.\lambda_{1}, \lambda_{2}, \cdots, \lambda_{r+1}\right)$ implies $F\left(\cdots, \bar{t}_{i_{1} i_{2}} \cdots i_{r}, \cdots\right)=0$.

3. Dependent base. When $r=k$ in $\S 2$, there results an $[n-k]$-congruence of order one in $[n]$ for which the fundamental variety on a generic $[n-k]$ is irreducible.

When $r<k$, other $[n-k]$-congruences of order one may be constructed as follows. For these, the fundamental variety on a generic $[n-k]$ is composite.

Consider

$$
\left\|\begin{array}{ccc}
x_{11}^{(2)} & \cdots & x_{s \delta+1}^{(2)} \\
\cdot & \cdots & \cdot \\
x_{81}^{(2)} & \cdots & x_{1 s+1}^{(2)}
\end{array}\right\|=0
$$

where $x_{i j}^{(2)} \equiv \sum_{k=0}^{n} a_{i j k}^{(2)} x_{k}$, in which $a_{i j k}^{(2)}$ are forms of order $\sigma_{21}$ (any positive integer or zero) in the coordinates $P_{h}(h=1,2, \cdots, r+1)$ of $(2.4)$.

(3.1) will be called a dependent base. Since this concept seems to be new, it is emphasized that the $x_{i j}^{(2)}$ are linear forms in the projective point coordinates of $[n]$, having coefficients which are forms of order $\sigma_{21}$ in the $P_{1}, P_{2}, \cdots, P_{r+1}$ of the $[n-r]$ through arbitrary $P$.

Geometrically, an arbitrary point $P$ of $[n]$ determines the $[n-r]$ of $(2.4)$ through it whose $P_{1}, \cdots, P_{r+1}$ make (3.1) a fixed base with elements that are linear forms in the running coordinates $x_{0}, \cdots, x_{n}$, with constant coefficients determined by $P_{1}, \cdots, P_{r+1}$. Thus there is defined in the $[n-r]$ through $P$ an $[n-r-s]$-congruence of order one whose $[n-r-s]$ through $P$ is given by equations (2.4) along with the equations

$$
\begin{aligned}
& P_{1}^{(2)} x_{11}^{(2)}+\cdots+P_{s+1}^{(2)} x_{1 s+1}^{(2)}=0 \\
& \cdot \cdot \cdot \cdot \cdot \cdot \cdot \cdot \cdot \cdot \cdot \\
& P_{1}^{(2)} x_{s 1}^{(2)}+\cdots+P_{s+1}^{(2)} x_{s s+1}^{(2)}=0
\end{aligned}
$$

in which $P_{j}^{(2)}$ is obtained by substituting the coordinates of $P$ in $X_{j}^{(2)}$, and $X_{j}^{(2)}$ is $(-1)^{j+1}$ times the determinant formed from the matrix of (3.1) by omitting the $j$ th column.

The ratios $P_{1}^{(2)}: P_{2}^{(2)}: \cdots: P_{s+1}^{(2)}$ are characteristic of the $[n-s]$ through $P$, given by (3.2). 
If $r+s=k$, equations (2.4) and (3.2) together define a unique $[n-k]$ through a generic point $P$ of $[n]$, and the totality of these $[n-k]$ 's constitute an $[n-k]$-congruence of order one in $[n]$. The fundamental variety on each $[n-k]$ of the congruence is composite, consisting of a $V_{n-k-1}^{r}$ in which the $[n-k]$ intersects (2.3) and a $V_{n-k-1}^{s}$ in which the $[n-k]$ intersects (3.2).

If $r+s<k$, another dependent base may be constructed whose elements are linear forms in $x_{0}, x_{1}, \cdots, x_{n}$ with coefficients that are homogeneous of order $\sigma_{31}$ in the $P_{1}, \cdots, P_{r+1}$ of (2.4) and also homogeneous of order $\sigma_{32}$ in the coordinates $P_{1}^{(2)}, \cdots, P_{s+1}^{(2)}$ of (3.2) $\left(\sigma_{31}\right.$ and $\sigma_{32}$ any positive integers or zero).

This process may be continued until the number of equations defining the generic flat space of the congruence is equal to $k$.

The effect of these dependent bases is to associate with each element of a flat space congruence of order one in $[n]$, in the most general possible manner, a unique congruence within that element.

4. Type $\left(A_{1} A_{2} \cdots A_{w}\right)_{n}$. We are now prepared to describe completely the general type $\left(A_{1} A_{2} \cdots A_{w}\right)_{n}$, where the $A_{i}$ are any positive integers whose sum is $k<n$.

Associated with the positive integer $A_{1}=r$ in first position in the type symbol is the fixed base

$$
\left\|\begin{array}{c}
x_{11} \cdots x_{1 r+1} \\
\cdots \\
x_{r 1} \cdots \\
x_{r r+1}
\end{array}\right\|=0
$$

in which the $x_{i j}$ are linear forms in $x_{0}, x_{1}, \cdots, x_{n}$ having arbitrary constant coefficients.

Then (\$2),

$$
\begin{aligned}
& P_{1} x_{11}+\cdots+P_{r+1} x_{1 r+1}=0, \\
& P_{1} x_{r 1}+\cdots+P_{r+1} x_{r+1}=0,
\end{aligned}
$$

where the $P_{i}$ are obtained by substituting the coordinates of an arbitrary point $P$ of $[n]$ in $X_{i}$, and $X_{i}$ is $(-1)^{i+1}$ times the determinant formed from the matrix of (4.1) by omitting the $i$ th column, represent an $[n-r]$ through $P$.

Associated with the positive integer $A_{h}=s$ in $h$ th position in the type symbol $(h=2,3, \cdots, w-1)$ is the dependent base

$$
\left\|\begin{array}{ccc}
x_{11}^{(h)} & \cdots & x_{18+1}^{(h)} \\
\cdot & \cdots & \cdot \\
x_{81}^{(h)} & \cdots & x_{88+1}^{(h)}
\end{array}\right\|=0,
$$

where $x_{i j}^{(h)} \equiv \sum_{k=0}^{n} a_{i j k}^{(h)} x_{k}$, in which $a_{i j k}^{(h)}$ are homogeneous of order $\sigma_{h 1}$ in the 
$P_{1}, \cdots, P_{r+1}$ of the flat space (4.2) through $P$, based on the fixed base (4.1) associated with $A_{1}$. The $a_{i j k}^{(h)}$ are also homogeneous of order $\sigma_{h m}$ in the $P_{1}^{(m)}, P_{2}^{(m)}, \cdots$ of the flat space through $P$ based on the dependent base associated with $A_{m}(m=2,3, \cdots, h-1)$. The $\sigma_{h 1}$ and $\sigma_{h m}$ are any positive integers or zero.

Then

$$
\begin{aligned}
& P_{1}^{(h)} x_{11}^{(h)}+\cdots+P_{s+1}^{(h)} x_{1 s+1}^{(h)}=0, \\
& \cdot \cdot \cdot \cdot \cdot \cdot \cdot \cdot \cdot \cdot \cdot \cdot \cdot \\
& P_{1}^{(h)} x_{s 1}^{(h)}+\cdots+P_{s+1}^{(h)} x_{s s+1}^{(h)}=0,
\end{aligned}
$$

where $P_{d}^{(h)}$ is the result obtained by substituting the coordinates of point $P$ in $X_{d}^{(h)}$, and $X_{d}^{(h)}$ is $(-1)^{d+1}$ times the determinant formed from the matrix of (4.3) by omitting the $d$ th column, represents an $[n-s]$ through $P$.

When the type symbol contains more than one integer, there is associated with the integer $A_{w}=t$, in last position in the type symbol, the terminal base

$$
\left\|\begin{array}{ccc}
x_{11}^{(w)} & \cdots & x_{1 t+1}^{(w)} \\
\cdot & \cdots & \cdot \\
x_{t 1}^{(w)} & \cdots & x_{t t+1}^{(w)}
\end{array}\right\|=0,
$$

where $x_{i j}^{(w)}=\sum_{k=0}^{n} a_{i j k}^{(w)} x_{k}$, in which $a_{i j k}^{(w)}$ are homogeneous of order $\tau_{j 1}$ in $P_{1}, P_{2}, \cdots$, homogeneous of order $\tau_{j 2}$ in $P_{1}^{(2)}, P_{2}^{(2)}, \cdots$, homogeneous of order $\tau_{j 3}$ in $P_{1}^{(3)}, P_{2}^{(3)}, \cdots$, and so on $(j=1,2, \cdots, t+1)$. The $\tau_{j k}$ are any positive integers or zero.

A terminal base differs from a dependent base in that the order of the forms comprising the coefficients of the linear forms in any particular column of the matrix of (4.5) may differ from the order of those in any other column. This additional generality is not ordinarily possible in the dependent base associated with $A_{h}$ for $h<w$, since it would cause loss of homogeneity in the $P_{d}^{(h+1)}$.

From (4.5) we have

$$
\begin{aligned}
& P_{1}^{(w)} x_{11}^{(w)}+\cdots+P_{t+1}^{(w)} x_{1 t+1}^{(w)}=0, \\
& P_{1}^{(w)} x_{t 1}^{(w)}+\cdots+P_{t+1}^{(w)} x_{t t+1}^{(w)}=0,
\end{aligned}
$$

in which the $P_{j}^{(w)}$ are obtained by substituting the coordinates of $P$ in $X_{j}^{(w)}$, and $X_{j}^{(w)}$ is $(-1)^{j+1}$ times the determinant formed by omitting the $j$ th column from the matrix of (4.5).

Equations (4.6) represent an $[n-t]$ through point $P$.

Equations (4.2), (4.4) (for $h=2,3, \cdots, w-1$ ) and (4.6) together represent a unique $[n-k]$ through an arbitrary point $P$ of $[n]$. The totality of 
such $[n-k]$ 's, for all points $\mathbb{P}_{\text {, of }}[n]$, form an $[n-k]$-congruence of order one in $[n]$, whose type symbol is $\left(A_{1} A_{2} \cdots A_{w}\right)_{n}$.

5. Fundamental points. For the $[n-k]$-congruence of order one in $[n]$ whose type symbol is $\left(A_{1} A_{2} \cdots A_{w}\right)_{n}$, there are $w$ varieties $\mathcal{A}_{1}, \mathcal{A}_{2}, \cdots, \mathcal{A}_{w}$, each of dimension $n-2$, all of whose points are fundamental. Through each point of any $\mathcal{A}_{i}$ there pass infinitely many $[n-k]$ 's.

$A_{1}$ is the fixed base (4.1). We have seen $(\$ 2)$ that $A_{1}$ is a variety of dimension $n-2$ and order $\alpha_{1}=C_{r+1,2}$.

The dependent base associated with $A_{2}$ of the type symbol is

$$
\left\|\begin{array}{ccc}
x_{11}^{(2)} & \cdots & x_{10+1}^{(2)} \\
\cdot & \cdots & \cdot \\
x_{s 1}^{(2)} & \cdots & x_{s s+1}^{(2)}
\end{array}\right\|=0
$$

where $x_{i j}^{(2)}$ are linear forms in $x_{0}, \cdots, x_{n}$, whose coefficients are forms of order $\sigma_{21}$ in $P_{1}, \cdots, P_{r+1}$.

Denote by $x_{i j}^{(2)}$ and $F_{j}^{(2)}$ the result of changing to $x_{0}, \cdots, x_{n}$ the coordinates of $P$ wherever they appear in $x_{i j}^{(2)}$ and $F_{j}^{(2)}$, respectively. Now

$$
\left\|\begin{array}{ccc}
{x_{11}^{(2)}}^{(2)} & x_{18+1}^{(2)} \\
\cdot & \cdots & \cdot \\
x_{81}^{(2)} & \cdots & x_{88+1}^{(2)}
\end{array}\right\|=0,
$$

is a variety of dimension $n-2$. Its order is the number of points in which it intersects a general plane of $[n]$. Since (5.2) has no special position relative to the frame of reference, its order will be given by the number of points in which it intersects the particular plane $\pi$, whose equations are $x_{3}=x_{4}=\cdots=x_{n}=0$. Since each element $x_{i j}^{(2)}$ of the matrix (5.2) is homogeneous of degree $\sigma_{21}$ in $F_{1}, \cdots, F_{r+1}$, every point of $A_{1}$ lies on (5.2). That is, (5.2) is a composite variety of dimension $n-2$, consisting of $A_{1}$ and a residual which is $\mathcal{A}_{2}$. We seek the order of $\mathcal{A}_{2}$. The order of $\mathcal{A}_{2}$ is the number of points, not on $\mathcal{A}_{1}$, in which the plane $\pi$ intersects (5.2).

Denote by $\xi_{i j}^{(2)}, \Phi_{j}$, and $\Phi_{j}^{(2)}$, the result obtained by equating to zero $x_{3}, x_{4}, \cdots, x_{n}$ in $x_{i j}^{(2)}, F_{j}$, and $F_{j}^{(2)}$, respectively. Salmon $\left({ }^{13}\right)$ has pointed out that the locus

$$
\left\|\begin{array}{ccc}
\xi_{11}^{(2)} & \cdots & \xi_{1 s+1}^{(2)} \\
\cdot & \cdots & \cdot \\
\xi_{s 1}^{(2)} & \cdots & \xi_{s s+1}^{(2)}
\end{array}\right\|=0
$$

(13) G. Salmon, Modern higher algebra, 4th edition, Dublin, 1885, p. 287. 
is the intersection of $\Phi_{1}^{(2)}=0$ and $\Phi_{2}^{(2)}=0$ after rejecting those points of the locus

$$
\left\|\begin{array}{ccc}
\xi_{13}^{(2)} & \cdots & \xi_{s 3}^{(2)} \\
\cdot & \cdots & \cdot \\
\xi_{1 s+1}^{(2)} & \cdots & \xi_{s s+1}^{(2)}
\end{array}\right\|=0
$$

which do not lie on $\Phi_{1}^{(2)}=0$ and $\Phi_{2}^{(2)}=0$. (Notice that the rows of this latter matrix are the columns of (5.3) common to $\Phi_{1}^{(2)}$ and $\Phi_{2}^{(2)}$.)

Let $s=1$ in (5.3). Then $\Phi_{1}^{(2)}=0$ is a plane curve of order $r \sigma_{21}+1$. Since $\Phi_{1}^{(2)}$ is homogeneous of degree $\sigma_{21}$ in $\Phi_{1}, \Phi_{2}, \cdots, \Phi_{r+1}$, and $\Phi_{1}=\Phi_{2}=\cdots=\Phi_{r+1}$ $=0$ is $C_{r+1,2}$ points, each of these points is $\sigma_{21}$-fold on the plane curve $\Phi_{1}^{(2)}=0$. Similarly, $\Phi_{2}^{(2)}=0$ is a plane curve of order $r \sigma_{21}+1$ on which each of the $C_{r+1,2}$ points, $\Phi_{1}=\Phi_{2}=\cdots=\Phi_{r+1}=0$, is $\sigma_{21}$-fold. Therefore $\Phi_{1}^{(2)}=0$ and $\Phi_{2}^{(2)}=0$ intersect in $\left(r \sigma_{21}+1\right)^{2}$ points, among which the $C_{r+1,2}$ points count $C_{r+1,2} \sigma_{21}^{2}$. That is, when $s=1$, the number of points, not on $\mathcal{A}_{1}$, in which (5.1) intersects $\pi$ is $\left(r \sigma_{21}+1\right)^{2}-C_{r+1,2} \sigma_{21}^{2}$. This is the order of $A_{2}$ when $s=1$.

Now consider the case $s=2$ in (5.3). $\Phi_{1}^{(2)}=0$ is a plane curve of order $2\left(r \sigma_{21}+1\right)$. Since $\Phi_{1}^{(2)}$ is homogeneous of degree $2 \sigma_{21}$ in $\Phi_{1}, \cdots, \Phi_{r+1}$, each of the $C_{r+1,2}$ points, $\Phi_{1}=\Phi_{2}=\cdots=\Phi_{r+1}=0$, is $2 \sigma_{21}$-fold on the plane curve $\Phi_{1}^{(2)}=0$. Similarly, $\Phi_{2}^{(2)}=0$ is a plane curve of order $2\left(r \sigma_{21}+1\right)$, on which each of the $C_{r+1,2}$ points is $2 \sigma_{21}$-fold. $\Phi_{1}^{(2)}=0$ and $\Phi_{2}^{(2)}=0$ intersect in $4\left(r \sigma_{21}+1\right)^{2}$ points in which the $C_{r+1,2}$ points count as $4 \sigma_{21}^{2} C_{r+1,2}$. That is, $\Phi_{1}^{(2)}=0$ and $\Phi_{2}^{(2)}=0$ intersect in $4\left(r \sigma_{21}+1\right)^{2}-4 \sigma_{21}^{2} C_{r+1,2}$ points not on $\mathcal{A}_{1}$. We have already seen $(s=1)$ that

$$
\left\|\xi_{13}^{(2)} \quad \xi_{23}^{(2)}\right\|=0
$$

consists of $\left(r \sigma_{21}+1\right)^{2}-\sigma_{21}^{2} C_{r+1,2}$ points not on $\mathcal{A}_{1}$. Therefore, for $s=2$, (5.3) consists of $3\left\{\left(r \sigma_{21}+1\right)^{2}-C_{r+1,2} \sigma_{21}^{2}\right\}$ points not on $\mathcal{A}_{1}$. This is the order of $\mathscr{A}_{2}$ for $s=2$.

By induction it can be shown that the order of $\mathcal{A}_{2}$, for $s$ any positive integer, is $\alpha_{2}=C_{s+1,2}\left\{\left(r \sigma_{21}+1\right)^{2}-C_{r+1,2} \sigma_{21}^{2}\right\}$.

The determination of the orders of $\mathcal{A}_{3}, \mathcal{A}_{4}, \cdots, \mathcal{A}_{w}$ becomes increasingly tedious and does not differ essentially from the above.

When $\sigma_{h k} \neq 0$ in the dependent base (4.3), the $P_{1}^{(k)}, P_{2}^{(k)}, \cdots$ appear homogeneously in the coefficients of the linear forms $x_{i j}^{(h)}$, and this causes $A_{h}$ to intersect $A_{k}$ in a variety of dimension $n-3(k<h)$. If, moreover, $\sigma_{k g} \neq 0$ $(g<k), \mathcal{A}_{k}$ intersects $\mathcal{A}_{o}$ in a variety of dimension $n-3$. But $P_{1}^{(o)}, P_{2}^{(o)}, \ldots$ appear in the $P_{1}^{(h)}, P_{2}^{(h)}, \cdots$, and therefore also in the coefficients of the linear forms $x_{i j}^{(h)}$, and consequently $\mathcal{A}_{h}$ intersects $\mathcal{A}_{0}$ in a variety of dimension $n-3$. If $\mathcal{A}_{k}$ intersects $\mathcal{A}_{0}$ in a variety of dimension $n-3$ and $\mathcal{A}_{h}$ intersects $\mathcal{A}_{k}$ in a variety of dimension $n-3(g<k<h)$, then $\mathcal{A}_{h}$ intersects $\mathcal{A}_{0}$ in a variety of dimension $n-3$. The three varieties of dimension $n-3$, just mentioned, are 
distinct. Subject only to this restriction, we cause $A_{h}$ to intersect $A_{g}$ in a $V_{n-3}$ or not, according as we choose $\sigma_{h o}$ different from, or equal to, zero in constructing our flat space congruence of type $\left(A_{1} \cdots A_{w}\right)_{n}$.

6. Coincident fundamental points. For convenience in the type symbol $\left(A_{1} A_{2} \cdots A_{h-1} A_{h} \cdots A_{w}\right)_{n}$, let $A_{1}+A_{2}+\cdots+A_{h-1}=u$. A unique $[n-u]$ of the congruence $\left(A_{1} A_{2} \cdots A_{h-1}\right)_{n}$ passes through $P$. This $[n-u]$ intersects $\mathcal{A}_{i}$ in $\mathcal{D}_{i}(i<h) . \mathscr{D}_{i}$ is a determinantal primal of space $[n-u]$ and is of dimension $n-u-1$ and order $A_{i}(\$ 2)$.

By means of the dependent base (4.3) there is determined in $[n-u]$ a fixed base $D$, whose matrix contains $s$ rows.

When $s=A_{i}(i<h), D$ can clearly lie on $\mathcal{A}_{i}$. When $s=A_{i}-1$, there are two systems of fixed bases, whose matrices have $s$ rows, on $\mathcal{D}_{i}\left({ }^{14}\right)$ and thus $D$ can lie on $\mathcal{D}_{i}$.

Therefore, whenever in the type symbol an integer is equal to, or one less than, an integer further to the left, there exists an $[n-k]$-congruence of order one in $[n]$ with coincidences among the fundamental points.

In the case of a line congruence having $A_{w}=1$ in its type symbol, the plane through $\mathrm{P}$ of the associated plane congruence $\left(A_{1} A_{2} \cdots A_{w-1}\right)_{n}$ intersects $A_{1}, \mathcal{A}_{2}, \cdots, \mathcal{A}_{w-1}$ in plane curves $\mathcal{D}_{1}, \mathcal{D}_{2}, \cdots, \mathcal{D}_{w-1}$, respectively, and $A_{w}$ in a single point. This point can be made to lie on any of the plane curves, and therefore for the type symbol $\left(A_{1} A_{2} \cdots A_{w-1} 1\right)_{n}$, with $A_{1}+A_{2}+\cdots$ $+A_{w-1}+1=n-1$, there exist line congruences of order one in $[n]$ whose fundamental points associated with $A_{w}$ are coincident with those associated with any earlier $A_{i}$.

7. Ordinary space. In the interest of exposition, the foregoing will now be applied to the well known situation in three-dimensional space.

There is only one plane congruence ${ }^{15}$ ) of order one in [3]. Its type symbol is $(1)_{3}$ and its fixed base is

$$
\left\|x_{11} \quad x_{12}\right\|=0
$$

where $x_{1 j}(j=1,2)$ are linear forms in $x_{0}, x_{1}, x_{2}, x_{3}$, with arbitrary constant coefficients.

(7.1) represents a fixed line in [3]. The unique plane through this line and a generic point $P$ is

$$
P_{1} x_{11}+P_{2} x_{12}=0,
$$

where $P_{1}$ is the result of substituting the coordinates of $P$ in $x_{12}$ and $P_{2}$ is the result of substituting the coordinates of $P$ in $-x_{11}$. The totality of planes (7.2) form a plane congruence of order one in [3].

The locus of fundamental points, $\mathcal{A}_{1}$, is the line (7.1).

(14) Room, loc. cit. p. 106.

$\left.{ }^{15}\right)$ While a pencil of planes in [3] is not ordinarily called a congruence, it fulfills our definition $(k=1)$, and such usage makes for uniformity of language. 
In [3] there are line congruences of order one with type symbols $(2)_{3}$ and $(11)_{3}$. Each gives rise to an additional line congruence having coincident fundamental points.

The fixed base for type $(2)_{3}$ is

$$
\left\|\begin{array}{lll}
x_{11} & x_{12} & x_{13} \\
x_{21} & x_{22} & x_{23}
\end{array}\right\|=0
$$

where the $x_{i j}$ are linear forms in $x_{0}, x_{1}, x_{2}, x_{3}$, having arbitrary constant coefficients. (7.3) is a twisted cubic curve whose unique bisecant through a generic point $P$ of $[3]$ is

$$
\begin{aligned}
& P_{1} x_{11}+P_{2} x_{12}+P_{3} x_{13}=0 \\
& P_{1} x_{21}+P_{2} x_{22}+P_{3} x_{23}=0 .
\end{aligned}
$$

The $P_{j}$ are obtained by substituting the coordinates of $P$ in $X_{j}(j=1,2,3)$, and $X_{j}$ is $(-1)^{j+1}$ times the determinant formed from the matrix of (7.3) by omitting the $j$ th column.

The totality of rays (7.4) is a line congruence of order one in [3] with type symbol $(2)_{3}$ and whose irreducible locus of fundamental points $\mathcal{A}_{1}$ is a twisted cubic curve (7.3).

If all the planes $x_{i j}=0(i=1,2 ; j=1,2,3)$ pass through the same point $O$, the twisted cubic (7.3) degenerates into three lines through $O$. The congruence now consists of the bundle of lines through $O$. The three lines (7.3) through $O$ are not fundamental but are members of the congruence. The only fundamental point is the vertex $O$. This oldest line congruence of order one in [3] is thus a special case of our type (2) . $_{3}$

In the line congruence of type $(11)_{3}$, the fixed base is the line (7.1). The terminal base is given by

$$
\left\|x_{11}^{(2)} \quad x_{12}^{(2)}\right\|=0
$$

where $x_{i j}^{(2)} \equiv \sum_{k=0}^{3} a_{i j k}^{(2)} x_{k}$, and $a_{1 j k}^{(2)}$ are forms of order $\tau_{j 1}$ (any positive integer or zero) in $P_{1}, P_{2}$ of plane (7.2). For each plane (7.2), (7.5) represents a line in [3] intersecting that plane in a point $O$.

The equation

$$
P_{1}^{(2)} x_{11}^{(2)}+P_{2}^{(2)} x_{12}^{(2)}=0,
$$

in which $P_{1}^{(2)}$ and $P_{2}^{(2)}$ are obtained by substituting the coordinates of $P$ for the running coordinates in $x_{12}^{(2)}$ and $-x_{11}^{(2)}$, respectively, represents for each $P_{1}: P_{2}$ a plane through $P$.

Equations (7.2) and (7.6) together represent the ray through $P$, lying in plane (7.2) and through point $O$. The totality of rays given by (7.2) and (7.6) form a line congruence of order one in [3].

The locus of fundamental points is composite, consisting of $\mathcal{A}_{1}$, the fixed 
line (7.1), and $\mathcal{A}_{2}$, a space curve of order $\tau_{11}+\tau_{21}+1$. The equations of $\mathcal{A}_{2}$ are formed from (7.5) by replacing the coordinates of $P$ in the $a_{1 j k}^{(2)}$ by the running coordinates $x_{0}, x_{1}, x_{2}, x_{3}$. These equations contain the line (7.1) counted $\tau_{11} \tau_{21}$ times. The space curve has $\tau_{11}+\tau_{21}$ intersections with line $\mathcal{A}_{1}$. Point $O$ is the intersection of curve $A_{2}$ with plane (7.2), not on $A_{1}$.

If, in the preceding type (11) ${ }_{3}$ congruence, we impose the restriction that the determinant of the coefficients of $x_{11}, x_{12}, x_{11}^{(2)}$, and $x_{12}^{(2)}$ shall vanish for all values of $P_{1}: P_{2}$, the two fundamental points on a generic ray of the congruence will coincide on the line (7.1). The locus of fundamental points is the line (7.1), provided that not both $\tau_{11}$ and $\tau_{21}$ are zero. There is established a $\left(\tau_{11}+\tau_{21}, 1\right)$ correspondence between the planes and points on (7.1). A generic point $P$ of [3] determines a plane through line (7.1) and this plane in turn determines one point $O$ on (7.1). Line $P O$ is the unique ray of the congruence through $P$.

It may be of interest to observe that while it has long been recognized that this latter congruence is a limiting case of the line congruence of type $(11)_{3}$, it has hitherto been thought impossible to obtain the coincident case by specialization of the parameters in the equations of type (11) 3 .

8. Aprile's congruence. The $[n-2]$ 's determined by the point groups of a $g_{n-1}^{n-1}$ belonging to a rational normal curve of order $2 n-3$ in $[2 n-3]$ form an $[n-2]$-congruence of order one in $[2 n-3]$. The intersections of the $[n-2]$ 's of this congruence with an $[n]$ of $[2 n-3]$ are lines of a line congruence of order one in $[n]$.

This construction is due to Aprile $\left({ }^{16}\right)$. We shall show that it is a restricted case of our type $(n-1)_{n}$ congruence.

Let the parametric equations of the normal rational curve $C$ in $[2 n-3]$ be $x_{i}=\lambda^{2 n-3-i} \mu^{i}(i=0,1,2, \cdots, 2 n-3)$.

Consider the fixed base

$$
\left\|\begin{array}{ccc}
x_{0} & x_{1} & x_{2} \cdots x_{n-1} \\
x_{1} & x_{2} & x_{3} \cdots x_{n} \\
\cdot & \cdot & \cdots \\
x_{n-2} & x_{n-1} & x_{n} \cdots x_{2 n-3}
\end{array}\right\|=0
$$

whose elements $x_{0}, x_{1}, \cdots, x_{2 n-3}$ are point coordinates in [2n-3]. Then

$$
\begin{aligned}
& P_{1} x_{0}+P_{2} x_{1}+\cdots+P_{n} x_{n-1}=0, \\
& P_{1} x_{1}+P_{2} x_{2}+\cdots+P_{n} x_{n}=0, \\
& \cdot \cdot \cdot \cdot \cdot \cdot \cdot \cdot \cdot \cdot \cdot \cdot \cdot \\
& P_{1} x_{n-2}+P_{2} x_{n-1}+\cdots+P_{n} x_{2 n-3}=0,
\end{aligned}
$$

(16) Aprile, loc. cit. p. 204. 
are the equations of a unique $[n-2]$ through a generic point $P$ of $[2 n-3]$.

(8.2) intersects the normal curve $C$ in the $n-1$ points whose parameters are given by

$$
P_{1} \lambda^{n-1}+P_{2} \lambda^{n-2} \mu+P_{3} \lambda^{n-3} \mu^{2}+\cdots+P_{n} \mu^{n-1}=0,
$$

as may be seen by substituting the parametric equations of curve $C$ in (8.2).

Thus Aprile's $[n-2]$-congruence in $[2 n-3]$ is the same as that given by (8.2), and the section of this congruence by an $[n]$ of $[2 n-3]$ is Aprile's line congruence of order one in $[n]$.

The $[n]$-section of $(8.1)$ is a fixed base. But (8.1) is what Room calls a partly symmetric determinantal locus, as is also its $[n]$-section. Hence $\left({ }^{17}\right)$, the freedom of the fixed base of Aprile's line congruence of order one in $[n]$ is

$$
\delta=(1 / 2)(n-1)(n-2)(n+1)-(n-1) n+1
$$

less than that of our fixed base, for $n>3$.

(17) Room, loc. cit. p. 160.

University of Arizona, Tucson, Ariz. 